\title{
A Novel Method for Solving Multiobjective Linear Programming Problems with Triangular Neutrosophic Numbers
}

\author{
Qing Wang $\mathbb{D}^{1},{ }^{1}$ Yi Huang, ${ }^{2,3}$ Shiming Kong, ${ }^{3}$ Xinqiang Ma $\mathbb{D},{ }^{2,3}$ Youyuan Liu, ${ }^{3}$ S. K. Das, ${ }^{4}$ \\ and S. A. Edalatpanah $\left(\mathbb{C}^{5}\right.$ \\ ${ }^{1}$ Department of Mathematics and Physics, Suzhou Vocational University, Suzhou 215104, China \\ ${ }^{2}$ College of Computer Science and Technology, Guizhou University, Guiyang, China \\ ${ }^{3}$ Institute of Intelligent Computing and Visualization Based on Big Data, Chongqing University of Arts and Sciences, \\ Chongqing, China \\ ${ }^{4}$ Department of Revenue, Ministry of Finance, Government of India, New Delhi, India \\ ${ }^{5}$ Department of Applied Mathematics, Ayandegan Institute of Higher Education, Tonekabon, Iran
}

Correspondence should be addressed to Qing Wang; wqing@jssvc.edu.cn and S. A. Edalatpanah; saedalatpanah@gmail.com

Received 6 November 2020; Revised 17 December 2020; Accepted 21 July 2021; Published 23 September 2021

Academic Editor: M M Bhatti

Copyright () 2021 Qing Wang et al. This is an open access article distributed under the Creative Commons Attribution License, which permits unrestricted use, distribution, and reproduction in any medium, provided the original work is properly cited.

In the field of operation research, linear programming (LP) is the most utilized apparatus for genuine application in various scales. In our genuine circumstances, the manager/decision-makers (DM) face problems to get the optimal solutions and it even sometimes becomes impossible. To overcome these limitations, neutrosophic set theory is presented, which can handle all types of decision, that is, concur, not certain, and differ, which is common in real-world situations. By thinking about these conditions, in this work, we introduced a method for solving neutrosophic multiobjective LP (NMOLP) problems having triangular neutrosophic numbers. In the literature study, there is no method for solving NMOLP problem. Therefore, here we consider a NMOLP problem with mixed constraints, where the parameters are assumed to be triangular neutrosophic numbers (TNNs). So, we propose a method for solving NMOLP problem with the help of linear membership function. After utilizing membership function, the problem is converted into equivalent crisp LP (CrLP) problem and solved by any suitable method which is readily available. To demonstrate the efficiency and accuracy of the proposed method, we consider one classical MOLP problem and solve it. Finally, we conclude that the proposed approach also helps decision-makers to not only know and optimize the most likely situation but also realize the outcomes in the optimistic and pessimistic business situations, so that decision-makers can prepare and take necessary actions for future uncertainty.

\section{Introduction}

Linear programming (LP) problem has an important application in various sectors of our daily life. The major drawback faced by manager or decision-makers (DM) in daily-life application is to determine the parameters. Because of several factors in real-life problems, the real-life problems are very complex. Due to uncertainty, the decision-makers cannot always formulate the problem in a well-defined and exact manner, nor can they always precisely predict the outcome of viable decisions. To overcome these uncertainty complex problems, we take more realistic descriptive knowledge of experts, which can be represented as fuzzy data. Firstly, the basic concept of fuzzy set theory was proposed by Zadeh [1]. Further, the basic concept of fuzzy decision-making was proposed by Bellman and Zadeh [2]. Thus, linear programming (LP) problem with fuzzy environment would be very effective in solving real-life problems. If the parameters of LP problem are considered as fuzzy, then it is called fuzzy linear programming (FLP) problem. The concepts of the feasible solution and $\alpha$ efficient solutions of the FLP problem were proposed by Ramik [3]. Maleki et al. introduced the idea of using the ranking function for solving the FLP problem [4]. The concept of sensitivity analysis for solving the FLP problem was proposed by Ebrahimnejad [5]. A trapezoidal fuzzy number was 
considered by Wan and Dong [6] for solving LP problems using multiobjective programming and membership function. Ganesan and Veeramani [7] also considered a new fuzzy symmetric trapezoidal fuzzy number and solved it. Another type of problem was considered by Lotfi et al. [8], where all the parameters, variables, and constraints are chosen as fully fuzzy LP (FFLP) problem and solved by lexicographic method. Kumar et al. [9] also proposed a method for solving FFLP problem with equality constraints by using ranking function. Najafi and Edalatpanah [10] have suggested some modifications of paper [9]. Many researchers [11-13] considered the lexicographic technique to apply in various problems like FFLP problem with triangular numbers and FFLP problem with trapezoidal fuzzy numbers.

After successful application of fuzzy sets in real-life application, decision-makers (DM) want a more realistic approach to handle the uncertainty in real-world problems. Thus, Atanassov [14] proposed the concept of a new set which is combined with both membership functions and nonmembership functions and the set was called intuitionistic fuzzy set (IFS). IFS is an extension version of fuzzy set. Singh and Yadav [15] proposed intuitionistic fuzzy multiobjective linear programming problem with various membership functions. Singh and Yadav [16] proposed transportation problem with intuitionistic fuzzy type-2 problem. Some of researchers [17-22] focused on solving multiobjective LP (MOLP) problem and LP problems with intuitionistic fuzzy numbers. Till now, several works have been pioneered in both FS and IFS. Afterwards, Smarandache [23] introduced the structure of neutrosophic set (NS) for developing the solution of any kind of real-world problem in a reasonable way. After Smarandache, Wang et al. [24] disclosed the establishment of single-typed neutrosophic set, which demands a crucial position in NS theory. The notion of single-valued neutrosophic set was more suitable for solving many reallife problems like image processing, medical diagnosis, decision-making, water resource management, and supply chain management. To reflect the decision-making information in an objective way, the triangular neutrosophic numbers (TNN) can be used in real-life problems to express the attribute value. This can not only maintain the variables but also highlight the possibility of various values within this interval. Of late, Abdel-Basset et al. [25] solved LP problems under neutrosophic triangular numbers by using ranking functions. An integer programming problem with triangular neutrosophic numbers was developed by Das and Edalatpanah [26]. For the first time in neutrosophic sets, Das and Chakraborty [27] proposed a model for solving LP problem in pentagonal numbers. Ye et al. [28] introduced the idea of finding the optimal solution of the LP problem in NNs environment. Das [29] also used pentagonal neutrosophic numbers in transportation problem. Pythagorean fuzzy numbers also can handle the uncertainly problem. Wang and Li [30] proposed a Pythagorean fuzzy number in decision-making problem; for more details about the applications of fuzzy extension sets, see [31-34].
Our contribution, motivation, and novelties are as follows.

1.1. Contribution. In this article, we mainly focused on neutrosophic multiobjective linear programming (NMOLP) problem with mixed constraints under triangular neutrosophic numbers. Several factors are also involved in our day-to-day life; therefore, DM choose the neutrosophic numbers for better results. In neutrosophic numbers, DM always choose any membership function as per the problem. Some basic operational laws of triangular neutrosophic numbers are demonstrated to enhance the pertinence of our proposed theory. With the progression of the study, a newly conceptualized ranking function is established under triangular neutrosophic number background. Utilizing this constructive tool, the NMOLP problem is transformed into crisp MOLP problem. Notably, the well-known various membership functions are used for conversion into an equivalent crisp convex programming problem.

1.2. Motivation. Neutrosophic sets play an important role in uncertainty modelling. The development of uncertainty theory plays a fundamental role in formulation of real-life scientific mathematical model, structural modelling in engineering field, medical diagnosis problem, and so forth. How can we solve multiobjective linear programming based triangular neutrosophic numbers? Is it possible to apply in real-life problem? Still there is no method for application in multiobjective linear programming problem having triangular neutrosophic numbers. From this aspect, we try to extend this research paper.

1.3. Novelties. A linear membership function is usually very comfortable in real-life situations. It is defined by two points, that is, the upper levels and lower levels of acceptability. A numerical problem related real mathematical problem is set forth to validate our anticipated hypothesis. Lastly, the comparison work involving the ranking system of the alternatives uplifted the superiority of our proposed supposition. To the best of our knowledge, no method is available for solving NMOLP problem. Therefore, we attempt to establish a new strategy to solve this problem.

The rest of the paper is organized as follows: Some basic definitions and preliminaries are presented in Section 2. In Section 3, the classical MOLP problem and membership functions are presented. The proposed method is discussed in Section 4. In Section 5, we present a numerical example, and a real-life problem is discussed. The analysis of the result is also discussed is Section 6. Finally, the conclusion is discussed in Section 7.

\section{Preliminaries}

In this segment, we establish some fundamental mathematical operations and definition which is required throughout the paper. 
Definition 1 (see [31]). Consider that $\widetilde{V}$ in all-inclusive discourse $X$, which is meant conventionally by $x$, is supposed to be a single-valued neutrosophic (SVN) set if $\widetilde{V}=\left\{\left\langle x:\left[\alpha^{p}(x), \alpha^{q}(x), \alpha^{r}(x)\right]\right\rangle: x \in X\right\}$. The set is described by a reality enrollment work, level of certainty: $\alpha^{P}(x): X \longrightarrow[0,1]$; an indeterminacy enrollment work, level of vulnerability: $\alpha^{q}(x): X \longrightarrow[0,1]$; and a false enrollment work, level of falsity: $\alpha^{r}(x): X \longrightarrow[0,1]$. Also, a SVN set satisfies the condition $0 \leq \alpha^{p}(x)+\alpha^{q}(x)+\alpha^{r}(x) \leq 3$.

Definition 2 (see [30]). A triangular neutrosophic number (TNN) is denoted by $\widetilde{V}=\left\langle\left(p^{l}, p^{m} p^{n}\right),\left(\alpha^{p}, \alpha^{q}, \alpha^{r}\right)\right\rangle$ whose three membership functions for the truth, indeterminacy, and falsity of $x$ can be defined as follows:

$$
\alpha^{p}(x)= \begin{cases}\frac{\left(x-p^{l}\right)}{\left(p^{m}-p^{n}\right)} \alpha^{p}, & p^{l} \leq x<p^{m}, \\ \alpha^{p}, & x=p^{l}, \\ \frac{\left(p^{n}-x\right)}{\left(p^{n}-p^{m}\right)} \alpha^{p}, & p^{m} \leq x<p^{n}, \\ 0, & \text { otherwise, } \\ \frac{\left(p^{m}-x\right)}{\left(p^{m}-p^{n}\right)} \alpha^{q}, & p^{l} \leq x<p^{m}, \\ \alpha^{q} & \\ \frac{\left(x-p^{n}\right)}{\alpha^{r},} \alpha^{q}, & p^{m} \leq x<p^{n}, \\ \frac{\left(p^{n}-x\right)}{\left(p^{m}-p^{n}\right)} \alpha^{r}, & p^{l} \leq x<p^{m}, \\ 1, & \text { otherwise, }\end{cases}
$$

where $0 \leq \alpha^{P}(x)+\alpha^{q}(x)+\alpha^{r}(x) \leq 3, x \in R$. Additionally, when $p^{l} \geq 0$, Ris called a nonnegative TNN. Similarly, when $p^{l}<0, R$ becomes a negative TNN.
Definition 3 (see[30]). Let $A_{1}=\left\langle\left(p^{l}, p^{m}, p^{n}\right),\left(\alpha^{p}, \alpha^{q}, \alpha^{r}\right)\right\rangle$ and $A_{2}=\left\langle\left(q^{l}, q^{m}, q^{n}\right),\left(\beta^{p}, \beta^{q}, \beta^{r}\right)\right\rangle$ be two TNNs. Then the arithmetic relations are defined as follows:

(i) $A_{1} \oplus A_{2}=\left\langle\left(p^{l}+q^{l}, p^{m}+q^{m}, p^{n}+p^{n}\right),\left(\alpha^{p} \wedge \beta^{p}\right.\right.$, $\left.\left.\alpha^{q} \wedge \beta^{q}, \alpha^{r} \wedge \beta^{r}\right)\right\rangle$.

(ii) $A_{1}-A_{2}=\left\langle\left(p^{l}-q^{n}, p^{m}-q^{m}, p^{n}-q^{l}\right),\left(\alpha^{p} \wedge \beta^{p}, \alpha^{q}\right.\right.$ $\left.\left.\wedge \beta^{q}, \alpha^{r} \wedge \beta^{r}\right)\right\rangle$.

(iii) $A_{1} \otimes A_{2}=\left\langle\left(p^{l} q^{l}, p^{m} q^{m}, p^{n} q^{n}\right),\left(\alpha^{p} \wedge \beta^{p}, \alpha^{q} \wedge \beta^{q}, \alpha^{r}\right.\right.$ $\left.\left.\wedge \beta^{r}\right)\right\rangle$, if $p^{-}>0, q^{l}>0,\left\langle\left(p^{l} q^{n}, p^{m} q^{m}, p^{n} q^{l}\right), \quad\left(\alpha^{p} \wedge\right.\right.$ $\left.\left.\beta^{p}, \alpha^{q} \wedge \beta^{q}, \alpha \quad{ }^{r} \wedge \beta^{r}\right)\right\rangle$, if $p^{l}<0, q^{l}>0,\left\langle\left(p^{n} q^{n}\right.\right.$, $\left.\left.p^{m} q^{m}, p^{l} q^{l}\right), \quad\left(\alpha^{p} \wedge \beta^{p}, \alpha^{q} \wedge \beta^{q}, \alpha^{r} \wedge \beta^{r}\right)\right\rangle$, if $p^{l}<0$, $q^{l}<0$.

(iv) $\lambda A_{1}=\left\{\left\langle\left(\lambda p^{l}, \lambda p^{m}, \lambda p^{n}\right), \quad\left(\alpha^{p}, \alpha^{q}, \alpha^{r}\right)\right\rangle\right.$, if $\left.\lambda\right\rangle$ $0,\left\langle\left(\lambda p^{n}, \lambda p^{m}, \lambda p^{l}\right),\left(\alpha^{p}, \alpha^{q}, \alpha^{r}\right)\right\rangle$, if $\lambda<0$.

(v) $A_{1} / A_{2}=\left\{\left\langle\left(p^{l} / q^{n}, p^{m} / q^{m}, \quad p^{n} / q^{l}\right) ; \alpha^{p} \wedge \beta^{p}, \alpha^{q} \wedge \beta^{q}\right.\right.$, $\left.\alpha^{r} \wedge \beta^{r}\right\rangle\left(p^{n}>0, q^{n}>0\right),\left\langle\left(p^{n} / \quad q^{n}, p^{m} / q^{m}, p^{l} / q^{l}\right)\right.$; $\left.\alpha^{p} \wedge \beta^{p}, \alpha^{q} \wedge \beta^{q}, \quad \alpha^{r} \wedge \beta^{r}\right\rangle\left(p^{n}<0, q^{n}>0\right),\left\langle\left(p^{n} / q^{l}\right.\right.$, $\left.p^{m} / q^{m}, p^{l} / q^{n}\right)$

$\alpha^{p} \wedge \beta^{p}, \alpha^{q} \wedge \beta^{q}, \alpha^{r} \wedge \beta^{r}>\left(p^{n}<0, q^{n}<0\right)$.

Definition 4 (see [30]). Ranking neutrosophic numbers is consistently assumed as a fundamental function in phonetic dynamic and some other neutrosophic application frameworks, which has been concentrated by numerous mathematicians. Separation measure between two neutrosophic numbers is firmly identified with the idea of neutrosophic numbers which is closely related to the concept of ranking neutrosophic numbers. Let $A_{1}=\left(p^{l}, p^{m}, p^{n}\right) ;\left(\alpha^{p}, \alpha^{q}, \alpha^{r}\right)$ be a TNN. The ranking function for triangular neutrosophic number $A_{1}$ is denoted by $\mathfrak{R}\left(A_{1}\right)$ and defined by $\mathfrak{R}\left(A_{1}\right)=\left(p^{l}+p^{m}+p^{n} / 9\right)\left(\alpha^{p}+\left(1-\alpha^{q}\right)+\left(1-\alpha^{r}\right)\right)$.

Definition 5 (see [29]). Let $A_{1}$ and $A_{2}$ be two TNNs. Let $A_{1}=\left\langle\left(p^{l}, p^{m}, p^{n}\right),\left(\alpha^{p}, \alpha^{q}, \alpha^{r}\right)\right\rangle$

and $A_{2}=\left\langle\left(q^{l}, q^{m}, q^{n}\right),\left(\beta^{p}, \beta^{q}, \beta^{r}\right)\right\rangle$ be two TNNs. Then, we have the following:

(i) $\mathfrak{R}\left(A_{1}\right) \leq \mathfrak{R}\left(A_{2}\right) \Longleftrightarrow A_{1} \leq A_{2}$

(ii) $\mathfrak{R}\left(A_{1}\right) \geq \mathfrak{R}\left(A_{2}\right) \Longleftrightarrow A_{1} \geq A_{2}$

(iii) $\mathfrak{R}\left(A_{1}\right)=\mathfrak{R}\left(A_{2}\right) \Longleftrightarrow A_{1}=A_{2}$

(iv) $\min \left(A_{1}, A_{2}\right)=A_{1}$, if $A_{1} \leq A_{2}$ or $A_{2} \geq A_{1}$

Definition 6. Let $A_{1}=\left\langle\left(p^{l}, p^{m}, p^{n}\right),\left(\alpha^{p}, \alpha^{q}, \alpha^{r}\right)\right\rangle \quad$ and $A_{2}=\left\langle\left(q^{l}, q^{m}, q^{n}\right),\left(\beta^{p}, \beta^{q}, \beta^{r}\right)\right\rangle$ be two TNNs; then $A_{1}=A_{2}$ if and only if $p^{l}=q^{l}, p^{m}=q^{m}, p^{r}=q^{r}, \alpha^{p}=\beta^{p}, \alpha^{q}=\beta^{q}$, and $\alpha^{r}=\beta^{r}$.

\section{Classical Problem of MOLP}

The popular multiobjective linear programming (MOLP) issue with blended requirements is portrayed by 


$$
\begin{array}{ll}
\operatorname{Min} & Z=\left[Z^{1}, Z^{2}, \ldots, Z^{L}\right] \\
& \sum_{h=1}^{o} b_{g h} y_{h} \geq d_{g}, g=1,2, \ldots, n_{1}, \\
\text { s.t. } \quad \sum_{h=1}^{o} b_{g h} y_{h} \leq d_{g}, g=1,2, \ldots, n_{2}, \\
\quad \sum_{h=1}^{o} b_{g h} y_{h}=d_{g}, g=1,2, \ldots, n_{3} \\
\quad y_{h} \geq 0, h=1,2, \ldots \ldots, o .
\end{array}
$$

Definition 7. Let $T_{q}$ be the doable district for (2). A point $\bar{y}$ is supposed to be productive or pareto ideal arrangement solution of (2) if there does not survive any $y \in T_{q}$ with end goal that $Z_{w}(\bar{y}) \geq Z_{w}(y) \forall w$ and $Z_{w}(\bar{y}) \geq Z_{w}(y)$ for any $w$.

Definition 8. A point $\tilde{y} \in T_{q}$ is supposed to be feeble pareto ideal arrangement of (2) if there does not survive any $y \in T_{q}$ with end goal $Z_{w}(\tilde{y}) \geq Z_{w}(y) \forall w$, where $w=1,2, \ldots \ldots, L$.

3.1. Membership Functions. There are different techniques to take care of a MOLP issue. These strategies are arranged into two general classes: scalarization techniques and nonscalarization techniques. These methodologies convert the MOLP issue into a solitary target programming problem. In the above literature study, we found that two types of membership functions are used for solving MOLP problem. Linear membership function is an emerging technique to solve fuzzy linear programming problem. Linear function is based on two points only, that is, upper level and lower level of acceptability of the decision variable. In uncertainty circumstances, this type of function is not fixed for all conditions. Therefore, here we considered both linear and nonlinear membership functions.

3.1.1. Linear Membership Functions. A linear membership function $\mu_{\mathrm{LM}}$ can be defined as follows:

$$
\mu_{\mathrm{LM}}\left(Z_{D}(x)\right)= \begin{cases}1, & \text { if } Z_{D} \leq L_{D}, \\ \frac{U_{D}-Z_{D}}{U_{D}-L_{D}}, & \text { if } L_{D} \leq Z_{D}<U_{D}, \\ 0, & \text { if } Z_{D} \geq U_{D} .\end{cases}
$$

3.1.2. Parabolic Membership Function. The parabolic membership function $\mu_{\mathrm{PM}}$ can be defined as follows:

$$
\mu_{\mathrm{PM}}\left(Z_{D}(x)\right)= \begin{cases}1, & \text { if } Z_{D} \leq L_{D} \\ 1-\frac{\left(Z_{D}-L_{D}\right)^{2}}{\left(U_{D}-L_{D}\right)^{2}}, & \text { if } L_{D} \leq Z_{D}<U_{D} \\ 0, & \text { if } Z_{D} \geq U_{D} .\end{cases}
$$

Corollary 1 (see [17]). The following sets where $\lambda \geq 0$ are convex sets:

$$
\begin{aligned}
& \left\{X: \mu_{L}\left(Z_{p}^{\prime}(X)\right) \geq \lambda\right\}, \\
& \left\{X: \mu_{H}\left(Z_{p}^{\prime}(X)\right) \geq \lambda, Z_{p}^{\prime}(X) \leq \frac{1}{2}\left(U_{p}+L_{p}\right)\right\}, \\
& \left\{X: \mu_{p}\left(Z_{p}^{\prime}(X)\right) \geq \lambda\right\} .
\end{aligned}
$$

Proof. The proof is straightforward.

\section{Proposed Method}

In this section, by using a new ranking function, we suggest a new method for solving NMOLP problems. The main work will be presented as follows:

Step 1: consider problem (2) of classical MOLP problem.

Step 2: on the off chance that the coefficients of the goal capacities, choice factors, and right-hand sides of requirements are dubious, which are spoken with by three-sided neutrosophic numbers, at that point problem (2) becomes NMOLP problem and the problem might be composed as

$$
\begin{array}{ll}
\operatorname{Min} & \widetilde{Z}_{N}=\left[\widetilde{Z}_{N}^{1}, \widetilde{Z}_{N}^{2}, \ldots, \widetilde{Z}_{N}^{L}\right] \\
& \sum_{h=1}^{o} \widetilde{b}_{g h}^{N} y_{j} \geq \widetilde{d}_{g}^{N}, g=1,2, \ldots \ldots, n_{1}, \\
\text { s.t. } \quad & \sum_{h=1}^{o} \widetilde{b}_{g h}^{N} y_{h} \leq \widetilde{d}_{g}^{N}, g=1,2, \ldots \ldots, n_{2}, \\
& \sum_{h=1}^{o} \widetilde{b}_{g h}^{N} y_{h}=\widetilde{d}_{g}^{N}, g=1,2, \ldots \ldots, n_{3}, \\
& y_{h} \geq 0, h=1,2, \ldots \ldots, o .
\end{array}
$$

Step 3: utilizing the ranking function which is linear, problem (7) is changed to the accompanying crisp MOLP problem. 


$$
\begin{array}{ll}
\operatorname{Min} & \widetilde{Z}=\left[\widetilde{Z}^{1}, \widetilde{Z}^{2}, \ldots, \widetilde{Z}^{L}\right] \\
& \sum_{h=1}^{o} \tilde{b}_{g h} y_{h} \geq \tilde{d}_{g}, g=1,2, \ldots \ldots, n_{1}, \\
& \quad \sum_{h=1}^{o} \tilde{b}_{g h} y_{h} \leq \tilde{d}_{g}, g=1,2, \ldots \ldots, n_{2}, \\
& \sum_{h=1}^{o} \tilde{b}_{g h} y_{h}=\tilde{d}_{g}, g=1,2, \ldots \ldots, n_{3}, \\
& y_{h} \geq 0, h=1,2, \ldots, \ldots .
\end{array}
$$

Step 4: determine the goal programming as follows:

$$
\begin{array}{ll}
\text { Find } & \left\{y_{h}, h=1,2, \ldots, o\right\} \\
& \widetilde{Z}_{D} \approx L_{D}, D=1,2, \ldots, L, \\
& \sum_{h=1}^{o} \widetilde{b}_{g h} y_{h} \geq \widetilde{d}_{g}, g=1,2, \ldots, n_{1}, \\
\text { s.t. } \quad & \sum_{h=1}^{o} \widetilde{b}_{g h} y_{h} \leq \widetilde{d}_{g}, g=1,2, \ldots, n_{2}, \\
& \sum_{h=1}^{o} \widetilde{b}_{g h} y_{h}=\widetilde{d}_{g}, g=1,2, \ldots, n_{3}, \\
& y_{h} \geq 0, h=1,2, \ldots, \ldots .
\end{array}
$$

In problem $(8)$, the symbol “ $\approx$ " is used to denote that some deviation ought to be permitted while exacting objective. To change the model in (8) into a crisp programming model, we already discussed the above linear and nonlinear membership functions.

Step 5: use appropriate enrollment works and change the GP model into crisp programming model.

Step 6: solve the crisp programming problem using any suitable technique or LINGO or MATLAB.

Theorem 1. An effective solution for (7) is a proficient solution for (6).

Proof. The proof is straightforward.

So, from the above theorem, NMOLP problem (6) is equal to settling crisp model (7).

\section{Numerical Experiments}

In this section, some numerical examples are given below to illustrate the new model.

Example 1. Let us consider the following NMOLP problem:

$$
\begin{aligned}
& \operatorname{Min} \widetilde{Z}_{N}^{1}=\widetilde{5}_{N} y_{1}+\widetilde{3}_{N} y_{2} \\
& \operatorname{Min} \widetilde{Z}_{N}^{2}=\widetilde{2}_{N} y_{1}+\widetilde{7}_{N} y_{2} \\
& \text { s.t. } \quad \widetilde{2}^{N} y_{1}+\widetilde{4}^{N} y_{2} \geq 2 \widetilde{5}^{N}, \widetilde{4}^{N} y_{1}+\widetilde{5}^{N} y_{2} \leq \widetilde{5} 0^{N}, \widetilde{1}^{N} y_{1} \\
& \quad+\widetilde{1}^{N} y_{2} \geq 1 \widetilde{0}^{N}, y_{1}, y_{2} \geq 0,
\end{aligned}
$$

where the parameters are as follows:

$$
\begin{aligned}
\widetilde{5} & =(4,5,6 ; 0.8,0.6,0.4), \\
\widetilde{3} & =(2.5,3,4 ; 0.75,0.5,0.3), \\
\widetilde{2} & =(2,2,3 ; 1,0.5,0), \\
\widetilde{7} & =(7,7,7.5 ; 0.8,0.6,0.4), \\
\widetilde{2} & =(1.5,2,2 ; 0.9,0.6,0.2), \\
\widetilde{4} & =(3,4,4 ; 1,0.5,0), \\
\widetilde{1} & =(0.5,1,1 ; 1,0.2,0.2), \\
\widetilde{1} & =(1,1,1 ; 1,0,0.5), \\
2 \widetilde{5} & =(22,25,25 ; 0.8,0.6,0.4), \\
1 \widetilde{0} & =(9,10,10 ; 1,0.5,0), \\
5 \widetilde{0} & =(50,50,55 ; 0.75,0.5,0.3) .
\end{aligned}
$$

As Step 3, we use ranking function of Definition 5; the above problem is equivalent to the following crisp MOLP problem:

$$
\begin{array}{ll}
\operatorname{Min} & \widetilde{Z}^{1}=3.001 y_{1}+2.058 y_{2} \\
\operatorname{Min} \widetilde{Z}^{2}=1.94 y_{1}+4.3 y_{2} \\
& 1.283 y_{1}+3.05 y_{2} \geq 14.4, \\
\text { s.t. } & 3.05 y_{1}+3.001 y_{2} \leq 30.14 \\
& 0.723 y_{1}+0.86 y_{2} \geq 8.05, \\
& y_{1}, y_{2} \geq 0 .
\end{array}
$$

Solving problem (11) as per Step 4, we have the following solutions:

$$
\begin{aligned}
y_{1} & =(0,8.721), \\
y_{2} & =(11.53,0.08), \\
L_{1} & =30.06 \\
U_{1} & =63.29 \\
L_{2} & =31.26 \\
U_{2} & =51.14
\end{aligned}
$$

where $L_{i}, U_{i}$ are deviation points of membership functions. Now, applying Step 5, problem (11) is equal to the accompanying GP model as follows:

$$
\begin{array}{ll}
\text { Find }\{ & \left.y_{h}: h=1,2\right\} \\
& 3.001 y_{1}+2.058 y_{2} \approx 30.06, \\
& 1.94 y_{1}+4.3 y_{2} \approx 31.26, \\
\text { s.t. } \quad & 1.283 y_{1}+3.05 y_{2} \geq 14.4, \\
& 3.05 y_{1}+3.001 y_{2} \leq 30.14, \\
& 0.723 y_{1}+0.86 y_{2} \geq 8.05, \\
& y_{1}, y_{2} \geq 0 .
\end{array}
$$

Applying the membership functions and solving by LINGO 18.0, the solution of (13) is reported in Table 1. 
TABle 1: Result discussion of Example 2.

\begin{tabular}{lcr}
\hline Membership functions & Solutions & Objective values \\
\hline Linear & $y_{1}=4.24, y_{2}=3.97$ & $(20.89,25.29 ; 0.8,0.6,0.4)$ \\
Parabolic & $y_{1}=5.32, y_{2}=4.07$ & $(24.14,27.82 ; 0.8,0.4,0.2)$ \\
\hline
\end{tabular}

Example 2. Let us consider the following NMOLP problem:

$$
\begin{array}{ll}
\operatorname{Max} & \widetilde{Z}_{N}^{1}=\widetilde{4}_{N} y_{1}+1 \widetilde{0}_{N} y_{2} \\
\operatorname{Max} & \widetilde{Z}_{N}^{2}=\widetilde{2}_{N} y_{1}+\widetilde{5}_{N} y_{2} \\
& \widetilde{2}^{N} y_{1}+y_{2} \leq \widetilde{5}^{N}, \\
\text { s.t. } & \widetilde{2}^{N} y_{1}+\widetilde{5}^{N} y_{2} \leq \widetilde{1}^{N}, \\
& y_{1}, y_{2} \geq 0 .
\end{array}
$$

Problem (14) can be modelled as a multiobjective neutrosophic linear programming problem with singlevalued triangular neutrosophic numbers.

$$
\begin{aligned}
& \operatorname{Max} \widetilde{Z}_{N}^{1}=(3,4,5 ; 0.5,0.5,0.6) y_{1}+(9,10,11 ; 0.5,0.7,0.4) y_{2} \\
& \operatorname{Max} \widetilde{Z}_{N}^{2}=(1,2,3 ; 0.5,0.5,0.5) y_{1}+(4,5,6 ; 0.5,0.7,0.4) y_{2} \\
& \qquad(1,2,3 ; 0.5,0.4,0.8) y_{1}+(1,1,1 ; 0.5,0.3,0.5) y_{2} \leq(4,5,6 ; 0.5,0.6,0.5)^{N}, \\
& \text { s.t. } \quad(1,2,3 ; 0.5,0.4,0.8) y_{1}+(4,5,6 ; 0.5,0.7,0.4) y_{2} \leq(9,10,11 ; 0.5,0.7,0.4)^{N}, \\
& \quad y_{1}, y_{2} \geq 0 .
\end{aligned}
$$

By utilizing our Step 3 to Step 6, the optimal solution of the above problem is reported in Table 2.

5.1. Real-Life Application: Diet Problem. In this section, to show the application of the proposed method, the real-life problem is solved by the proposed method, and it is concluded that the proposed method can be applied in any reallife problem. For a very simple diet problem in which the nutrients are starch and protein as a group, the two types of foods with data are given in Table 3.
The activities and their levels in the model are given as follows: activity $j$ : to include $1 \mathrm{~kg}$ of food type $j$ in the diet, associated level $y_{j}$, for $j=1,2$. The various nutrients in the model lead to different constraints. For example, the amount of starch contained in the diet is $5 y_{1}+2 y_{2}$, which must be $\geq 5$ for feasibility. Similarly, $y_{1}+2 y_{2} \geq 6$. In this diet problem, the total cost of food and the procurement cost of food should be minimized. Since the cost coefficients and all other coefficients are indecisive and also contain the indeterminacy part, the problem is modelled as a bilevel multiobjective linear programming problem.

The formulation of the above problem is given as follows:

$$
\begin{aligned}
& \operatorname{Min} \widetilde{Z}_{N}^{1}=(2,3,4, ; 0.6,0.5,0.5) y_{1}+(1,1,1 ; 0.5,0.7,0.5) y_{2} \\
& \operatorname{Min} \widetilde{Z}_{N}^{2}=(1,2,3 ; 0.6,0.5,0.5) y_{1}+(2,3,4 ; 0.6,0.5,0.5) y_{2} \\
& \qquad(4,5,6 ; 0.6,0.5,0.5) y_{1}+(1,2,3 ; 0.5,0.7,0.5) y_{2} \geq(4,5,6 ; 0.6,0.5,0.5)^{N}, \\
& \text { s.t. } \quad(1,1,1 ; 0.5,0.7,0.5) y_{1}+(1,2,3 ; 0.5,0.7,0.5) y_{2} \geq(5,6,7 ; 0.5,0.6,0.5)^{N}, \\
& \quad y_{1}, y_{2} \geq 0 .
\end{aligned}
$$


TABLE 2: Result discussion of Example 2.

\begin{tabular}{lcc}
\hline Membership functions & Solutions & Objective values \\
\hline Linear & $y_{1}=1.87, y_{2}=1.25$ & $(18.26,19.98,25.43 ; 0.5,0.7,0.6)$ \\
Parabolic & $y_{1}=1.58, y_{2}=1.52$ & $(5.4,8.5,13 ; 0.5,0.7,0.6)$ \\
\hline
\end{tabular}

TABLE 3: Data considered by the manager.

\begin{tabular}{lccc}
\hline \multirow{2}{*}{ Nutrients } & \multicolumn{4}{c}{ Nutrient units/kg of food type } \\
& Food 1 & Food 2 & Minimum requirements \\
\hline Starch & 5 & 1 & 5 \\
Protein & 2 & 2 & 6 \\
Cost $/ \mathrm{kg}$ & 3 & 1 & \\
Procurement cost $/ \mathrm{kg}$ & 2 & 3 & \\
\hline
\end{tabular}

TABLE 4: Results of diet problem.

\begin{tabular}{lcc}
\hline $\begin{array}{l}\text { Membership } \\
\text { functions }\end{array}$ & Solutions & Objective values \\
\hline $\begin{array}{l}\text { Linear } \\
\text { Parabolic }\end{array}$ & $y_{1}=0.5, y_{2}=2.5$ & $(3.5,4,4.5 ; 0.6,0.5,0.5)$ \\
$y_{1}=3, y_{2}=0$ & $(3,6,9 ; 0.5,0.7,0.5)$ \\
\hline
\end{tabular}

TABLE 5: Comparison of the proposed method with the existing method [17].

\begin{tabular}{lcc}
\hline Membership functions & Proposed method & Existing method [17] \\
\hline Linear & 4 & 4.8 \\
Parabolic & 6 & 7
\end{tabular}

Using our method, the above problem is solved using our Step 3 to Step 5, and the above optimal solution is obtained in Table 4.

\section{Result Analysis}

In the above literature study, we found that there is no method for solving multiobjective linear programming problem in neutrosophic environment. Therefore, for rationality and effectiveness of the proposed method, we consider another uncertainty problem, that is, intuitionistic fuzzy numbers. Singh and Yadav [17] considered the same problem and solved it with various membership functions. Here, we consider the diet problem for comparison of our proposed method with the existing method.

From Table 5, we get that our result is better than the existing results. Since the object of the problem is minimization, so based on this point of view, our results are better than the existing approach under both linear and parabolic membership functions. Therefore, we can conclude that our proposed algorithm is a new way to handle the uncertainty in real-life problems.

\section{Conclusions}

In this paper, we develop a new method for solving neutrosophic multiobjective LP (NMOLP) problems, and the model is transformed into a MOLP problem by using ranking function. After successful application in ranking function, we use scalarization technique to convert the goal programming (GP) problem. We also investigated various membership functions to solve the GP model. As per our discussion, the DM choose the membership functions independently which fit the model. From the obtained results, we conclude that the nonlinear membership functions, that is, parabolic functions, are always better than linear membership functions (parabolic $>$ linear). To the best of our knowledge, there is no method in literature for solving NMOLP problem by using membership functions. We also used our proposed method to demonstrate a numerical example. Our proposed method is a new way in neutrosophic environment to handle multiobjective programming problem. There are various scopes in the future to develop our algorithm, like application in real-life problem from industrial sector, transportation problem, and assignment problem.

\section{Data Availability}

The data used to support the findings of this study are included within the article.

\section{Conflicts of Interest}

The authors declare no conflicts of interest.

\section{Authors' Contributions}

The authors contributed equally to writing this article. All authors have read and agreed to the published version of the manuscript.

\section{Acknowledgments}

This research was funded by the Key Industrial Technology Development Project of Chongqing Development and Reform Commission, China (Grant no. 2018148208), Key Technological Innovation and Application Development Project of Chongqing, China (Grant no. cstc2019jscxfxydX0094), Innovation and Entrepreneurship Demonstration Team of Yingcai Program of Chongqing, China (Grant no. CQYC201903167), and Science and Technology Innovation Project of Yongchuan District (Grant no. Ycstc, 2020cc0501). The authors express their gratitude for the support.

\section{References}

[1] L. A. Zadeh, "Fuzzy sets," Information and Control, vol. 8, no. 3, pp. 338-353, 1965. 
[2] R. E. Bellman and L. A. Zadeh, "Decision-making in a fuzzy environment," Management Science, vol. 17, no. 4, 141 pages, 1970.

[3] J. Ramík, "Duality in fuzzy linear programming: some new concepts and results," Fuzzy Optimization and Decision Making, vol. 4, no. 1, pp. 25-39, 2005.

[4] H. R. Maleki, M. Tata, and M. Mashinchi, "Linear programming with fuzzy variables," Fuzzy Sets and Systems, vol. 109, no. 1, pp. 21-33, 2000.

[5] A. Ebrahimnejad and M. Tavana, "A novel method for solving linear programming problems with symmetric trapezoidal fuzzy numbers," Applied Mathematical Modelling, vol. 38, no. 17-18, pp. 4388-4395, 2014.

[6] S. P. Wan and J. Y. Dong, "Possibility linear programming with trapezoidal fuzzy numbers," Applied Mathematical Modelling, vol. 38, no. 5-6, pp. 1660-1672, 2014.

[7] K. Ganesan and P. Veeramani, "Fuzzy linear programs with trapezoidal fuzzy numbers," Annals of Operations Research, vol. 143 , no. 1 , pp. 305-315, 2006.

[8] F. Hosseinzadeh Lotfi, T. Allahviranloo, M. Alimardani Jondabeh, and L. Alizadeh, "Solving a full fuzzy linear programming using lexicography method and fuzzy approximate solution," Applied Mathematical Modelling, vol. 33, no. 7, pp. 3151-3156, 2009.

[9] A. Kumar, J. Kaur, and P. Singh, "A new method for solving fully fuzzy linear programming problems," Applied Mathematical Modelling, vol. 35, no. 2, pp. 817-823, 2011.

[10] H. S. Najafi and S. A. Edalatpanah, "A note on "A new method for solving fully fuzzy linear programming problems"” $A p$ plied Mathematical Modelling, vol. 37, no. 14-15, pp. 78657867, 2013.

[11] M. Dehghan, B. Hashemi, and M. Ghatee, "Computational methods for solving fully fuzzy linear systems," Applied Mathematics and Computation, vol. 179, no. 1, pp. 328-343, 2006.

[12] R. Ezzati, E. Khorram, and R. Enayati, “A new algorithm to solve fully fuzzy linear programming problems using the MOLP problem," Applied Mathematical Modelling, vol. 39, no. 12, pp. 3183-3193, 2015.

[13] B. Pérez-Cañedo and E. R. Concepción-Morales, "A method to find the unique optimal fuzzy value of fully fuzzy linear programming problems with inequality constraints having unrestricted L-R fuzzy parameters and decision variables," Expert Systems with Applications, vol. 123, pp. 256-269, 2019.

[14] K. T. Atanssov, "Intuitionistic fuzzy set," Fuzzy Sets and Systems, vol. 20, pp. 87-96, 1986.

[15] S. K. Singh and S. P. Yadav, "Intuitionistic fuzzy multi-objective linear programming problem with various membership functions," Annals of Operations Research, vol. 269, no. 12, pp. 693-707, 2018.

[16] S. K. Singh and S. P. Yadav, "A new approach for solving intuitionistic fuzzy transportation problem of type-2," Annals of Operations Research, vol. 243, no. 1-2, pp. 349-363, 2016.

[17] B. Jana and T. K. Roy, "Multi-objective intuitionistic fuzzy linear programming and its application in transportation model," Notes on Intuitionistic Fuzzy Sets, vol. 13, no. 1, pp. 34-51, 2007.

[18] B. Jana and T. K. Roy, "Multi-objective fuzzy linear programming and its application in transportation model," Tamsui Oxford Journal of Mathematical Sciences, vol. 21, no. 2, pp. 243-269, 2005.

[19] R. Parvathi and C. Malathi, "Intuitionistic fuzzy linear programming problems," World Applied Sciences Journal, vol. 17, no. 12, pp. 1802-1807, 2012.
[20] D. Dubey and A. Mehra, "Linear programming with triangular intuitionistic fuzzy number," in Proceedings of the 7th Conference of the European Society for Fuzzy Logic and Technology, pp. 563-569, Atlantis Press, August 2011.

[21] S. K.Bharati and S. R. Singh, "A note on solving a fully intuitionistic fuzzy linear programming problem based on sign distance," International Journal of Computer Applications, vol. 119, no. 23, pp. 30-35, 2015.

[22] S. K. Bharati and S. R. Singh, "Solving multi objective linear programming problems using intuitionistic fuzzy optimization method: a comparative study," International Journal of Modeling and Optimization, vol. 4, no. 1, pp. 10-16, 2014.

[23] F. Smarandache, "Neutrosophy: neutrosophic probability, set, and logic: analytic synthesis \& synthetic analysis," 1998.

[24] H. Wang, F. Smarandache, Y. Zhang, and R. Sunderraman, "Single valued neutrosophic sets," Multispace and Multistruct, vol. 4, pp. 410-413, 2010.

[25] M. Abdel-Basset, M. Gunasekaran, M. Mohamed, and F. Smarandache, "A novel method for solving the fully neutrosophic linear programming problems," Neural Computing and Applications, vol. 31, no. 5, pp. 1595-1605, 2019.

[26] S. K. Das and S. A. Edalatpanah, "A new ranking function of triangular neutrosophic number and its application in integer programming," International Journal of Neutrosophic Science, vol. 4, no. 2, pp. 82-92, 2020.

[27] S. K. Das and A. Chakraborty, "A new approach to evaluate linear programming problem in pentagonal neutrosophic environment," Complex \& Intelligent Systems, vol. 7, no. 1, pp. 101-110, 2020.

[28] J. Ye, "Neutrosophic number linear programming method and its application under neutrosophic number environments," Soft Computing, vol. 22, no. 14, pp. 4639-4646, 2018.

[29] S. Kumar Das, "Application of transportation problem under pentagonal Neutrosophic environment," Journal of Fuzzy Extension and Applications, vol. 1, no. 1, pp. 27-41, 2020.

[30] L. Wang and N. Li, "Pythagorean fuzzy interaction power Bonferroni mean aggregation operators in multiple attribute decision making," International Journal of Intelligent Systems, vol. 35, no. 1, pp. 150-183, 2020.

[31] İ. Deli, "A novel defuzzification method of SV-trapezoidal neutrosophic numbers and multi-attribute decision making: a comparative analysis," Soft Computing, vol. 23, no. 23, pp. 12529-12545, 2019.

[32] A. Saha, I. Deli, and S. Broumi, "Hesitant triangular neutrosophic numbers and their applications to MADM," Neutrosophic Sets and Systems, vol. 35, pp. 269-298, 2020.

[33] M. Riaz and S. T. Tehrim, "On bipolar fuzzy soft topology with decision-making," Soft Computing, vol. 24, no. 24, pp. 18259-18272, 2020.

[34] N. Martin, R. Priya, and F. Smarandache, "New plithogenic sub cognitive maps approach with mediating effects of factors in COVID-19 diagnostic model," Journal of Fuzzy Extension and Applications, vol. 2, no. 1, pp. 1-14, 2021. 